\title{
A formação audiovisual dos educadores: A educação audiovisual nas graduações em pedagogia no Brasil $^{1}$
}

\author{
Audiovisual education training: Audiovisual education in \\ undergraduate courses in pedagogy in Brazil
}

\section{Formación en educación audiovisual: \\ Educación audiovisual en cursos de grado en pedagogía en Brasil}

\author{
Rafael Romão Silva \\ Universidade Federal Fluminense - UFF - Rio de Janeiro - Brasil \\ ORCID: https://orcid.org/0000-0002-9010-2603 \\ Endereço currículo Plataforma Lattes: http://lattes.cnpq.br/3239637898038719 \\ E-mail: rafael.romaos@gmail.com
}

Resumo: O Audiovisual se consolida no século XXI como experiência educativa. Seja como material didático, suporte de criação ou objeto de análise, ele passou a permear as experiências educativas em todos os níveis de ensino. Diversas áreas do conhecimento realizaram experiências e acumularam reflexões sobre as possíveis interfaces entre Audiovisual e Educação, dentre elas: Comunicação, Cinema, Arte e Educação. A partir desta diversidade metodológica, identificamos quais delas permeiam as dez melhores graduações de Licenciatura em Pedagogia do Brasil de acordo com o Ministério da Educação do Brasil, o que nos dá indícios de como os futuros docentes e gestores enxergam a Escola atravessada pelo Audiovisual.

Palavras-chave: Pedagogia. Educação Audiovisual. Educomunicação. Cinema- Educação. Educação a distância.

\begin{abstract}
Audiovisual is consolidated in the 21st century as an educational experience. Whether as didactic material, creative support or object of analysis, it began to permeate educational experiences at all levels of education. Several areas of knowledge have experimented and accumulated reflections on the possible interfaces between Audiovisual and Education, among them: Communication, Cinema, Arts and Education. Based on this methodological diversity, we have identified which of them permeate the ten best undergraduate degrees in Pedagogy in Brazil according to the Ministry of Education of Brazil, which gives us indications of how future teachers and managers see the School crossed by Audiovisual.
\end{abstract}

\footnotetext{
${ }^{1}$ Trabalho foi apresentado no XXIII Encontro da Sociedade Brasileira de Estudos de Cinema e Audiovisual em 2019, com resumo expandido publicado nos anais.
} 
Key words: Pedagogy. Audiovisual Education. Educommunication. Cine-Education. Distance learning.

Resumén: El audiovisual se consolida en el siglo XXI como una experiencia educativa. Ya sea como material didáctico, apoyo creativo u objeto de análisis, comenzó a impregnar las experiencias educativas en todos los niveles de la educación. Varias áreas de conocimiento llevaron a cabo experiencias y reflexiones acumuladas sobre las posibles interfaces entre Audiovisual y Educación, entre ellas: Comunicación, Cine, Arte y Educación. En base a esta diversidad metodológica, hemos identificado cuáles de ellos impregnan los diez mejores cursos de grado en Pedagogía en Brasil según el Ministerio de Educación de Brasil, lo que nos da indicaciones de cómo los futuros maestros y gerentes ven a la Escuela atravesada por Audiovisual.

Palabras-llave: Pedagogía. Educación audiovisual. Educomunicación. Cine-educación. Educación a distancia.

\section{A educação audiovisual e suas expressões}

São quatro as principais áreas do conhecimento que realizaram experiências e pensam a Educação em face ao Audiovisual no Brasil entre os séculos XX e XXI, áreas por sua vez institucionalizadas enquanto departamentos universitários e cursos de pós-graduação. São elas: o Cinema, a Comunicação, a Arte e a Pedagogia. Uma busca pela história do pensamento brasileiro revela várias terminações que surgiram nestas áreas: comunicação educacional, mídia-educação, cinema-educação, comunicação/educação, educomunicação, educação midiática etc. Ainda, o pensamento sobre as TICS coloca essa reflexão dentro da interface telecomunicações/educação e ao lado de outras tecnologias, como a informática.

A constatação desta diversidade é importante para a formação do educador, seja o próprio educador audiovisual (MACHADO, 2018), quanto o educador que agora convive com as telas. Porém, ambos certamente se perdem em meio a tantas identidades. É reivindicado o uso do termo Educação Audiovisual ${ }^{2}$, proposto como uma forma de

\footnotetext{
${ }^{2}$ Sérgio Alberto Rizzo Junior e Moira Toledo Cirello escreveram teses de doutorado utilizando este termo.
} DOI: http://doi.org/10.14393/par-v5n1-2020-53796 - Paradoxos, Uberlândia, v. 5, n. 1, p. 86-100, jan./jun. 2020 | 87 


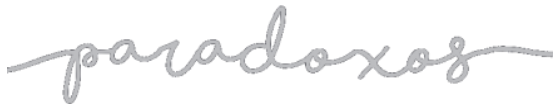

A formação audiovisual dos educadores:

A educação audiovisual nas graduações em Pedagogia no Brasil

SILVA, 2020

reconhecer essas ramificações, diálogo com as experiências encontradas pela história que pode contribuir para um processo de formação complexa, diversa e dialógica.

Assim, dada a importância e o tamanho de uma área tão particular ao nosso tempo, o objetivo do presente artigo é refletir sobre como as diferentes abordagens da Educação Audiovisual ocorre dentro da formação de professores. Para tanto, é realizada uma análise bibliográfica que alcança as graduações de Pedagogia, com foco na inserção do Audiovisual dentro de cada curso de graduação. O conjunto de documentos abordados envolve as Diretrizes Curriculares Nacionais para o Curso de Graduação de Licenciatura em Pedagogia (BRASIL, 2006) e os projetos políticos-pedagógicos dos dez melhores cursos de Pedagogia em universidades públicas brasileiras de acordo com o Conceito Preliminar de Curso, publicado em 2017 pelo INEP, por sua vez vinculado ao Ministério da Educação do Brasil (INEP, 2019). Neste estudo são consideradas as dez melhores graduações de licenciatura em Pedagogia: Licenciatura em Pedagogia na modalidade a distância das Universidades Federal de São Carlos e da Universidade de Brasília e na modalidade presencial da Universidade do Estado de São Paulo campus São José do Rio Preto, Universidade da Integração Internacional da Lusofonia AfroBrasileira, Universidade Estadual do Centro-Oeste do Paraná campus Irati, Faculdade da Fundação Educacional Araçatuba, Instituto Federal de Goiás campus Goiânia Oeste, Universidade Federal de São Carlos campus Sorocaba, Instituto Federal Goiano e Universidade Federal Rural de Pernambuco.

A restrição por um conjunto de cursos em universidades públicas envolve a garantia da disponibilização de documentos para acesso público, o que não ocorre dentro das organizações privadas. Por fim, vemos como válida a escolha pelo conjunto das dez melhores graduações por elas possivelmente se transformarem em modelos para outras graduações do país. A restrição por cursos de Pedagogia envolve a acepção de que este tipo de curso forma profissionais que transversalmente atravessam a Escola, tanto na sala de aula, quanto na formação de outros professores e na elaboração e implementação de políticas públicas.

A análise dos documentos é feita a partir da busca por termos que indicam a possível presença da Educação Audiovisual dentro da proposta formativa, sendo eles: Cinema, Audiovisual, Filme, Comunicação, Vídeo, Educomunicação, Mídia, Tecnologia. 


\section{Os múltiplos atravessamentos entre a escola e o audiovisual}

Antes de entrar nas análises dos documentos eleitos, são apresentadas algumas das possíveis formas em que o Audiovisual pode estar presente na Escola e nos processos de ensino-aprendizagem. Para refletir sobre estas múltiplas possibilidades, é rastreado como as proposições de Educação Audiovisual são realizadas pelos quatro campos do conhecimento destacados por esta pesquisa: Cinema, Comunicação, Arte e Pedagogia.

O Cinema e o filme atravessaram a escola e os processos de ensino-aprendizagem de múltiplas formas nos últimos anos. Uma primeira forma envolve a produção de obras diretamente para as escolas ou para o processo educativo escolar ou não-escolar. O INCE (Instituto Nacional de Cinema Educativo) atuou nesta direção. Criado 1936 pelo Estado Novo, foi a primeira instituição nacional para o Cinema, um estúdio cinematográfico destinado à produção de filmes chamados educativos, à distribuição destes filmes e à criação de estrutura de exibição em escolas. Diversas pesquisas abordaram a produção cinematográfica do INCE, dentre elas a de Rosana Catelli (2005).

Uma segunda relação entre Cinema e Educação envolve a exibição de todo e qualquer filme, o que é reconhecer a potência educativa do audiovisual em si. Assim os filmes documentários, experimentais, ficções e animações podem ensinar sobre o mundo, as emoções, a história, o próprio cinema etc. Esses filmes podem ser projetados na escola, o que transforma as paredes e salas em espelhos e janelas para diferentes universos, ou a escola pode ir ao cinema, o que também gera seus próprios significados. Ainda, os cineclubes povoaram as escolas durante o século, o que fomentou a cultura cinematográfica dentro e fora dos currículos escolares.

A terceira forma envolve que a produção do filme ocorra na própria escola com a comunidade escolar. Isto também pode ser feito de várias maneiras, seja como uma simulação do modo de produção clássico, seja segundo as perspectivas do documentário contemporâneo, com a comunidade escolar enquanto equipe realizadora ou enquanto objeto de filmagem.

Na relação entre Educação e Comunicação, Ismar Oliveira Soares identifica três protocolos básicos, "entendidos como conjuntos de conceitos e normas que garantem a identidade das ações, sua coerência e aceitação pública: o moral, o cultural e o mediático (ou educomunicativo)" (SOARES, 2014, p. 17). São protocolos que abrigaram as práticas educativas com diversas mídias, dentre elas o audiovisual. O protocolo moral tem origens em 
movimentos religiosos e impulsionou construções sociais como o Código Hays $^{3}$ e a classificação indicativa. O protocolo moral parte dos pressupostos da responsabilidade social e da proteção da juventude e infância contra os excessos midiáticos. É uma abordagem que posteriormente se estende a movimentos laicos da sociedade, próximo a discussões sobre práticas de defesa ideológica. O segundo protocolo, o cultural, parte do pressuposto de que a comunicação faz parte da cultura e por isso deve ser estudada. No âmbito internacional se consolida enquanto Media Literacy. "O que caracteriza esta vertente é seu foco na relação dos educandos com os meios de comunicação e as novas tecnologias ou, simplesmente, com a mídia" (SOARES, 2014, p. 18). Já o protocolo mediático (sic) reivindica que a Media Education "deixasse de ser vista como um problema meramente educativo para transformarse num problema de natureza cultural" (SOARES, 2014, p. 22). A partir do acúmulo latinoamericano com as experiências de comunicação comunitária, a Educomunicação, nome que as práticas assumiram em torno deste protocolo, é uma abordagem que tira o privilégio das mídias enquanto objetos de estudo e práticas para focar na capacidade de expressão das crianças e jovens. "Na escola, o que se propõe é a revisão das disfunções comunicativas oriundas das relações de poder, buscando-se formas democráticas e participativas da gestão escolar, com o envolvimento de novas gerações” (SOARES, 2014, p. 18).

Quanto à Arte, temos o Audiovisual disperso entre as quatro linguagens que compõem o currículo de Arte no Ensino Básico. A maior parte delas é fundamentada pela Arte-Educação de Ana Mae Barbosa (2010), que com sua abordagem triangular trouxe a reflexão metodológica sobre ver, fazer e refletir dentro dos processos de ensino-aprendizagem. Também encontramos dentro desta interface os processos que lidam com objetos audiovisuais e práticas que não são considerados dentro do campo do Cinema. O trabalho de Greice Cohn (2016) dentro de uma reflexão sobre as pedagogias da videoarte é um exemplo desta perspectiva.

O audiovisual tem encontrado processos educativos na Arte também fora dos espaços formais de ensino. É o caso da dupla de artistas Maurício Dias e Walter Riedweg (VELOSO, 2012), que possui vários trabalhos com vídeos e instalações. Um destes é "Serviços Internos" (1995), que Velloso relata ter sido construído com 280

\footnotetext{
3 “(...) campanha com um abaixo-assinado de 10 milhões de assinaturas obrigou Hollywood a adotar regras de conduta (Código Hays), comprometendo-se a controlar os excessos denunciados por esta vertente" (SOARES, 2014, p. 17).
}

DOI: http://doi.org/10.14393/par-v5n1-2020-53796 - Paradoxos, Uberlândia, v. 5, n. 1, p. 86-100, jan./jun. 2020 | 90 


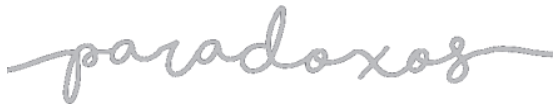

A formação audiovisual dos educadores:

A educação audiovisual nas graduações em Pedagogia no Brasil

SILVA, 2020

jovens estrangeiros recém-chegados na Suíça, onde participaram de classes de acolhimento dentro do sistema público de educação.

Por fim, na Pedagogia, o audiovisual tem sido objeto de reflexões alarmadas sobre como os meios de comunicação podem ameaçar ou não as escolas e professores. José Carlos Libâneo foi um dos autores nacionais que buscou apaziguar o eventual pânico e pavimentar caminhos para a construção de propostas. Em "Adeus professor, adeus professora? Novas exigências educacionais e profissão docente" (1998) ele busca construir uma perspectiva que reúna a formação crítica e social com as demandas do mercado em um contexto globalizado. Isso o leva a reconhecer que as Novas Tecnologias da Comunicação e Informação constituem o conjunto de mediações culturais que caracterizam o ensino. A perspectiva de Libâneo desemboca nas abordadas anteriores, porém muitos educadores desenvolveram seus próprios percursos dentro de suas áreas de conhecimento, nos quais construíram planos de aula e projetos que trazem o audiovisual para a sala de aula.

O audiovisual também é uma preocupação da Pedagogia dentro dos cursos em modalidade a distância, nos quais ele é um canal para a própria experiência educativa. Existem diferentes tipos de vídeos produzidos especificamente com propósitos de ensinoaprendizagem. A videoaula é o modelo mais utilizado, recebendo diferentes abordagens que passam pelo modelo de talking heads, incorporam técnicas de dramaturgia e também animações. Outro modelo de audiovisual destinado para processos de ensinoaprendizagem é o do videoensaio. Por fim, outros produtos audiovisuais passam a atravessar o processo educativo. É o caso dos videogames e seus serious games e as experiências em realidade virtual e aumentada.

\section{A Educação Audiovisual nos cursos de Pedagogia}

Como estas diferentes abordagens se manifestam nos cursos de graduação em Pedagogia? Um primeiro sinal destas efetuações está nas Diretrizes Curriculares Nacionais para os cursos de Pedagogia. Segundo Leda Scheibe (2007), as diretrizes para cursos de graduação no Brasil são criadas pelas Comissões de Especialistas de Ensino, sujeitas a posterior aprovação pelo Conselho Nacional de Educação. Scheibe relata todo o processo de negociação em torno da criação das diretrizes para as graduações em Pedagogia, o que envolveu o interesse acadêmico de que elas fossem afastadas do modelo 
das Escolas Normais. Assim se consolidou uma proposta que tem a prática docente como base, considera a gestão e pesquisa como atribuições do Licenciado em Pedagogia e propõe como diretriz a flexibilização dos conteúdos.

Para além de uma abordagem que pressupõe o Audiovisual dentro da disciplina de Artes ou enquanto um material didático para qualquer conteúdo, é o item VII do artigo $5^{\circ}$ que contempla uma das propostas da Educação Audiovisual nas Diretrizes Curriculares Nacionais.

\footnotetext{
Art. $5^{\circ} \mathrm{O}$ egresso do curso de Pedagogia deverá estar apto a: (...) VII relacionar as linguagens dos meios de comunicação à educação, nos processos didático-pedagógicos, demonstrando domínio das tecnologias de informação e comunicação adequadas ao desenvolvimento de aprendizagens significativas. (BRASIL, 2006, p. 2)
}

A partir deste item, é possível identificar que as diretrizes abordam a Comunicação enquanto meios de comunicação, o que já afasta a proposta da Educomunicação, ou seja, ignora o reconhecimento do fenômeno comunicativo dentro dos processos de ensino-aprendizagem. O foco se dá sobre os aspectos de linguagem desses meios, o que abre brechas para uma abordagem que não passa pelo potencial expressivo do audiovisual, mas foca na linguagem consolidada pelos meios de comunicação de massa. Portanto é possível identificar que essa diretriz está dentro do Protocolo Cultural da Comunicação e Educação, conforme apresentado acima dentro das reflexões de Ismar Oliveira.

Porém, cada Graduação em Pedagogia tem uma margem de liberdade sobre como interpretar as Diretrizes Curriculares e efetuá-las em um Projeto Político Pedagógico (PPP). Portanto, foi pesquisado os Projetos Político-Pedagógico das dez melhores graduações de Licenciatura em Pedagogia do país. Das dez melhores graduações do país, três delas não possuem os PPP's em seus respectivos websites: UNICENTRO, IF Goiano e FEA Araçatuba, sendo elas, então, excluídas das análises deste trabalho.

Outro ponto preliminar envolve a própria data de criação dos PPP's dentro de cada universidade. Conforme a tabela 1 indica, apenas a Licenciatura em pedagogia da UNESP teve o seu documento criado em 2001, antes do implantamento das diretrizes nacionais. Todas as outras tiveram seu PPP criado após 2006, sendo que a maior parte delas (5) o fez na década de 2010 (quadro 1). 
QUADRO 1 - Data de criação dos PPPs das 10 melhores graduações em Pedagogia do Brasil

\begin{tabular}{|c|c|c|c|}
\hline Universidade & Modalidade & Campus & Ano de criação do PPP \\
\hline UNESP & Presencial & São José do Rio Preto & 2001 \\
\hline UFRPE & Presencial & Garanhuns & 2006 \\
\hline UFSCar & EAD & -- & 2010 \\
\hline UNB & EAD & -- & 2011 \\
\hline UFSCAR & Presencial & Sorocaba & 2014 \\
\hline UNILAB & Presencial & Palmares & 2016 \\
\hline IFG & Presencial & Goiânia Oeste & N/D \\
\hline FAC FEA & Presencial & Araçatuba & N/D \\
\hline IF Goiano & Presencial & Morrinhos & N/D \\
\hline UNICENTRO & Presencial & Irati & \\
\hline
\end{tabular}

Fonte: Produção do próprio autor.

Através dos PPP's realizamos a busca dos conceitos selecionados como chaves de análise dentro de nossa pesquisa: Cinema, Audiovisual, Filme, Comunicação, Vídeo, Educomunicação, Mídia e Tecnologia. O quadro 2 indica em quais PPPs cada termo foi encontrado.

QUADRO 2 - Conceitos da educação audiovisual encontrados nos PPPs dos cursos de Pedagogia

\begin{tabular}{|c|c|c|c|}
\hline Universidade & Modalidade & Campus & Conceitos encontrados \\
\hline UNESP & Presencial & São José do Rio Preto & Comunicação, Mídia, Tecnologia, Vídeo \\
\hline UFRPE & Presencial & Garanhuns & Comunicação, Mídia, Tecnologia, Vídeo \\
\hline UFSCar & EAD & -- & Audiovisual, Comunicação, Mídia, Tecnologia \\
\hline UNB & EAD & -- & Comunicação, Mídia, Tecnologia, Vídeo \\
\hline UFSCAR & Presencial & Sorocaba & Comunicação, Educomunicação, Tecnologia \\
\hline UNILAB & Presencial & Palmares & $\begin{array}{l}\text { Audiovisual,Cinema, Comunicação, Mídia, } \\
\text { Tecnologia, Vídeo }\end{array}$ \\
\hline IFG & Presencial & Goiânia Oeste & $\begin{array}{l}\text { Audiovisual, Comunicação, Cinema, Mídia, } \\
\text { Tecnologia, Vídeo }\end{array}$ \\
\hline
\end{tabular}

Fonte: Produção do próprio autor. 
Há a presença do conceito de Comunicação, Tecnologia e Vídeo em quase todos os cursos. Chama a atenção a relativa ausência de conceitos como Filme e Educomunicação, o que dá indícios de que esses conceitos podem não ganhar destaque dentro das acepções de Comunicação que são trabalhados a partir dos PPPs.

No PPP da UNESP, o conceito de "Comunicação" é indicado da mesma forma que nas Diretrizes Curriculares Nacionais, o que mostra uma afinidade com elas, embora o PPP seja de cinco anos antes. Indicado enquanto "atribuições inerentes ao exercício profissional" (UNIVERSIDADE ESTADUAL PAULISTA, 2001, p. 35), o educador deve ser capaz de associar as linguagens dos meios de comunicação ao processo educativo. O conceito de "Tecnologia" recebe um tratamento genérico, sendo citado como necessário que os processos de ensino-aprendizagem se apropriem das TICs. Porém, nenhum desses conceitos atravessa os programas das disciplinas do curso. O mesmo ocorre com o conceito de "Mídia", ou seja, é apenas indicado que ele é importante para a formação dos pedagogos, mas não atravessa as disciplinas.

Já na UFRPE há a incidência de termos como "Vídeo", que emerge dentro da disciplina "Metodologia do Ensino da Geografia II", único componente curricular que explicita a criação de materiais didáticos multimidiáticos. Ainda, o audiovisual é citado dentro do projeto de Laboratório de Ensino, espaço dedicado para "viabilizar a Instrumentação para o Ensino, com a realização de experiências diversas (...), a realização de vídeo-conferências (...) e permitir a edição de programas educativos" (UNIVERSIDADE FEDERAL RURAL DE PERNAMBUCO, 2006, p. 74). A "Mídia” surge enquanto uma das linhas de pesquisa que o curso abriga, especificamente a "Educação e Tecnologias Multimidiáticas". Já a "Comunicação" emerge no projeto de acordo com as Diretrizes Curriculares Nacionais, o que também ocorre com "Tecnologia", e dentro do projeto de "Núcleos de estudos integradores", que tem como propósito estimular práticas de comunicação e expressão cultural. Dentro das disciplinas, a Comunicação é citada apenas em "Metodologia do Estudo e Produção Textual I", componente que tem o objetivo de estimular a prática textual acadêmica. Quanto à Tecnologia, duas disciplinas a abordam: "Informática em Educação I" e "Informática em Educação II", que tem como objetivos uma discussão crítica sobre o tema, noções básicas sobre o computador, ferramentas para fins pedagógicos, produção de sites, levantamento de dados para pesquisas e os ambientes virtuais de aprendizagem. 
No projeto da UFSCar Sorocaba (UNIVERSIDADE FEDERAL DE SÃO CARLOS, 2010) a "Comunicação" também emerge de acordo com as Diretrizes Curriculares, enquanto um meio a ser dominado pelo professor para uma posterior adequação ao processo educativo. Tal visão se concretiza no currículo através da disciplina "Educação, Comunicação e Tecnologias", que leva em seu programa a discussão sobre novas linguagens, seus impactos nas trocas globais e as concepções e práticas na Educomunicação em si. Já a dimensão tecnológica desta disciplina envolve as transformações operadas no pensamento pela tecnologia da informação. A Comunicação também é pensada enquanto um fenômeno em si na disciplina "Pensamento, Linguagem e Desenvolvimento Humano", que conta com uma bibliografia com foco nos trabalhos de Jean Piaget e Lev Vygotski.

Na graduação a distância (EaD) da UFSCar (UNIVERSIDADE FEDERAL DE SÃO CARLOS, 2010) encontramos o “Audiovisual” enquanto um material didático presente em seu Ambiente Virtual de Aprendizagem (AVA). Nada é dito sobre a sua forma, nem sobre uma possível integração entre a sua produção e o currículo do curso. É indicado que o curso conte com uma equipe produtora de obras audiovisuais para contextos educativos. A mesma instrumentalização ocorre com o conceito de "Mídia". Já o conceito de "Comunicação" atravessa todo o projeto do curso, seja como uma preocupação dentro do AVA, um ponto a ser desenvolvido entre a Instituição de Ensino Superior, seus polos e a comunidade externa e enquanto uma habilidade necessária aos professores e tutores dos polos do curso. A "Comunicação" também atravessa algumas disciplinas obrigatórias do curso: "Educação a Distância: Instrumentos e Tecnologias", que busca posicionar a EaD em suas bases históricas, metodológicas e instrumentais e "Letramento digital", que busca apresentar a estrutura e as possibilidades de uso dos AVAs, dos computadores e de alguns softwares. A Comunicação atravessa outras duas disciplinas optativas, disciplinas que buscam instrumentalizar os educadores para a Comunicação: "Língua Portuguesa: teoria e prática" e "Libras". Já o conceito de "Tecnologia" aparece enquanto um eixo que está presente na estrutura do curso, mas não além da abordagem utilitária que encontramos para o conceito de "Comunicação". Quanto às disciplinas de "Linguagens: Artes 1" e “Linguagens: Artes 2", o Cinema e o Audiovisual não são abordados em nenhuma delas, sendo a primeira voltada para a Música e a segunda para a Fotografia. Embora a Fotografia seja um dos elementos que compõem o Audiovisual, a Música e qualquer outra Arte 
também o são, o que não nos permite identificar a Fotografia enquanto a presença do Audiovisual na composição do curso.

Já no curso a distância da UnB (UNIVERSIDADE DE BRASÍLIA, 2011) o conceito de "Comunicação" e o de "Tecnologia" também são explicitados dentro de um panorama instrumental. "Um desafio complementar no novo currículo é o da formação para e pelo uso das tecnologias da informação e da comunicação (TIC) em educação". Esta perspectiva atravessa explicitamente o programa de duas disciplinas: "Educação para o trabalho" e "Educação de Jovens e Adultos", nas quais o programa da disciplina é discutido em situações "mediadas ou não pelas tecnologias da informação e comunicação". Uma única disciplina aborda efetivamente o conceito de Comunicação, a disciplina "Educação a distância" e ela o faz através de uma restrição ao discutir a Comunicação apenas no âmbito da EAD. O conceito de "Vídeo" é citado de forma instrumental, similar ao de "Audiovisual" enquanto meio para a educação a distância no PPP da UFSCar, ou seja, ele é apenas listado como um dos materiais didáticos disponíveis no curso. Já o conceito de "Tecnologia" aparece em uma disciplina chamada "Ensino de Ciências e Tecnologia I", na qual são discutidos a sua definição e abordagens didáticas para a tecnologia no Ensino Fundamental I. Também são citadas possíveis "Oficinas de Tecnologia" que possam ocorrer durante a trajetória acadêmica, embora sem um modelo específico indicado dentro do documento. O curso da UnB explicita a instrumentalização da Comunicação e Tecnologia para os processos de ensino-aprendizagem. Porém, parece que esta reflexão se restringe aos processos da EAD, o que eles propõem que ocorra principalmente através do estudante enquanto um usuário da EAD.

No PPP da UNILAB o conceito de "Cinema" surge enquanto uma das atividades válidas enquanto Atividade Complementar. Com a carga horária máxima de 100 horas, organizar mostras e festivais de Cinema e fazer cursos sobre esta arte é visto como complementar à formação do licenciado em Pedagogia. O mesmo ocorre com o conceito de "Audiovisual", o qual é considerado uma Atividade Complementar se o discente produzir uma exposição individual ou coletiva com obras de tal tipo. Quanto ao "Vídeo", na UNILAB ele é um dos tipos de Trabalho de Conclusão de Curso (TCC) que são aceitos, o qual deve ser entregue junto de uma produção textual que o embase. O conceito de "Comunicação" surge no projeto da UNILAB enquanto algo inerente ao projeto de fomentar a cultura afro-brasileira. No resgate das epistemologias negras, a Comunicação 


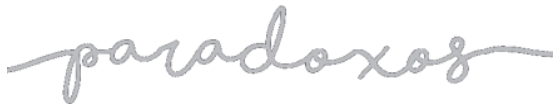

A formação audiovisual dos educadores:

A educação audiovisual nas graduações em Pedagogia no Brasil

SILVA, 2020

está dentro dos "conceitos e teorias [que] são imprescindíveis no processo de reconhecimento das sistematizações oriundas da África e da Diáspora" (UNIVERSIDADE DA INTEGRAÇÃO INTERNACIONAL DA LUSOFONIA AFROBRASILEIRA, 2016, p. 20). A Comunicação surge dentro do projeto de interdisciplinaridade empreendido pelo curso e como ferramentas que devem ser apropriadas em um mundo de trocas globais. Dentro das disciplinas, a Comunicação surge apenas dentro dos conceitos de "Fundamentos da Educação Especial e Inclusiva". Sobre o conceito de "Tecnologia", o PPP da UNILAB lhe dá o mesmo tratamento que ao de Comunicação, isto é, é citado que a apropriação das TICs é uma das linhas de formação do curso, mas a Tecnologia só surge dentro da disciplina focada em Educação Especial.

Já no IFG campus Goiânia Oeste o conceito de "Cinema” aparece como um objeto de seu Laboratório de Ensino, espaço destinado para a experimentação pedagógica que atravessa um conjunto de disciplinas. No caso do Cinema, ele aparece destacado na sua interface com a área de História, na qual ele é um dos objetos do qual o pedagogo deve ter "a capacidade de compreender, criticar e historicizar informações em diversos tipos de fontes" (INSTITUTO FEDERAL DE EDUCAÇÃO, CIÊNCIA E TECNOLOGIA DE GOIÁS, 2017, p. 13). O “Audiovisual” também é citado junto ao "Vídeo" como um dos recursos disponíveis em "Fundamentos e Metodologias do Ensino da Língua Portuguesa". O projeto cita a existência de um cargo técnico na área de Audiovisual, embora não se explicite sobre as formas de sua atuação dentro do curso. Sobre a "Mídia", as mídias sociais são apresentadas enquanto o único exemplo de tecnologia que deve ser contemplada na formação do Educador Social, linha de formação que atravessa o projeto do curso. Uma disciplina também contempla o conceito de Mídia: "Educação, Mídias e Tecnologias digitais” que tem na bibliografia obras como “O que é mídia-educação?” (2001) de Maria Luiza Belloni. Sua ementa contempla: "Fundamentos teórico-metodológicos das relações entre as tecnologias e a educação. Processos formativos mediados pelas tecnologias digitais. Educação em rede, mídias digitais e formação de professores". A “Tecnologia" também aparece na disciplina "Ciência, Tecnologia e Sociedade", disciplina que busca se aproximar de forma complexa dos conceitos que a intitula. 


\section{Conclusão}

Temos consciência de que a experiência universitária está além do que é descrito pelos PPPs. Porém, tudo isto está no campo da possibilidade, o que muitas vezes posiciona de forma irregular as oportunidades no caminho do corpo discente. Professores saem de licença, aposentam-se, mudam a abordagem de suas disciplinas, iniciam e encerram projetos. Os PPPs são, portanto, uma representação da experiência de parte dos discentes nas graduações.

A análise dos documentos eleitos para este estudo nos ajuda a entender dois pontos: i. como se concretizam na formação de pedagogos as reflexões sobre as interfaces entre TICS e Educação; ii. o descompasso entre o estado da reflexão sobre a Educação Audiovisual e as tendências identificadas dentro dos PPPs dos cursos.

Cabe ressaltar que todos os projetos indicam a comunicação e as tecnologias como um eixo relevante para a formação do pedagogo. No que tange a consolidação desta perspectiva nas disciplinas, apenas uma das graduações não possui nenhuma disciplina que explicite atravessamentos com a Educação Audiovisual (UNESP). Uma outra a tem unicamente dentro da disciplina de Educação Especial (UNILAB), que pensa o fenômeno comunicacional em sua amplitude para além das mídias e sua linguagem hegemônica.

Nas outras cinco graduações há disciplinas de diferentes perfis que apresentam diversos recortes dentro das interfaces que as telas podem ter com a Educação. Nelas, há tanto disciplinas voltadas para o próprio universo da Educação Audiovisual, quanto outras que a abordam em relação a outros componentes curriculares. Quatro delas abordam a Comunicação em si dentro de disciplinas obrigatórias (UFSCar Sorocaba, UFSCar EaD, UNB EaD e IFG) e três delas abordam a Tecnologia em si (UFRPE, UNB EaD e UFSCar EaD). Apenas uma das graduações presenciais efetivamente aborda a Informática como um componente curricular, já que na maioria dos outros cursos há uma preferência pela simbiose das TICS.

Já com o Audiovisual como objeto em si, não há a incidência de nenhuma disciplina que o aborde, a não ser "Linguagens II: Arte" da UFSCar EAD que aborda o tema da Fotografia. Também observamos que o Audiovisual emerge nos PPPs como um tópico aplicado em outras áreas em apenas uma das graduações em Pedagogia, dentro das reflexões sobre o ensino de Geografia na UFRPE. Porém, ele é citado enquanto objeto de projetos de extensão (UFRPE), enquanto material didático disponível dentro de AVAs (UFSCar EaD e UNB), como atividade complementar 


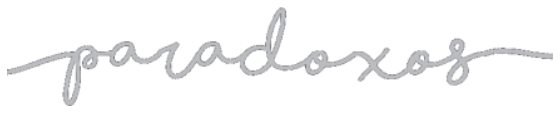

A formação audiovisual dos educadores:

A educação audiovisual nas

graduações em Pedagogia no Brasil

SILVA, 2020

permitida para a formação extra do discente (UNILAB) e como mídia de trabalho de conclusão de curso que o licenciando pode realizar para se graduar (UNILAB).

Por fim, as TICS e as mídias como um todo, citadas de forma genérica, também aparecem aplicadas a algumas disciplinas (Educação para o Trabalho e Educação de Adultos na UNB) e enquanto linhas de pesquisa dentro da graduação (UFRPE).

São muitas as possibilidades de o Audiovisual entrar na formação dos pedagogos. Com o presente artigo, demonstramos essa diversidade, o que pode abrir caminhos para refletir sobre como a Educação pode encarar as telas na educação sob formas mais complexas.

\section{Referências}

BARBOSA, Ana Mae; Cunha, Fernanda Pereira da (Orgs.). A abordagem triangular no ensino das artes e culturas visuais. São Paulo: Cortez. 2010.

BERGALA, Alain. A hipótese-cinema: pequeno tratado de transmissão do cinema dentro e fora da escola. Rio de Janeiro: Booklink, 2008.

BRASIL. Conselho Nacional de Educação. Parecer CNE/CP 5, 13.12.2005. Brasília, 2005.

BRASIL. Conselho Nacional de Educação. Resolução n.1, 15.5.2006. Diário Oficial da União, n.92, seção 1, p. 1112, mai. 2006.

CATELLI, Rosana Elisa. O cinema educativo nos anos de 1920 e 1930: algumas tendências presentes na biografia contemporânea. São Paulo: Intexto (UFRGS), 2005.

COHN, Grace. Pedagogias da videoarte: a experiência do encontro de estudantes do Colégio Pedro II com obras contemporâneas. 2016. Tese (Doutorado em Educação), Faculdade de Educação. Universidade Federal do Rio de Janeiro.

DUARTE, Rosália. Cinema \& educação. Belo Horizonte: Autêntica, 2002. 126p.

INSTITUTO FEDERAL DE EDUCAÇÃO, CIÊNCIA E TECNOLOGIA DE GOIÁS. Projeto Pedagógico (PP): Curso de Licenciatura em Pedagogia. 2017.

INSTITUTO FEDERAL DE EDUCAÇÃO, CIÊNCIA E TECNOLOGIA GOIANO. Licenciatura em pedagogia. Acesso em: 13 fev. 2019.

INSTITUTO NACIONAL DE ESTUDOS E PESQUISAS EDUCACIONAIS ANÍSIO TEIXEIRA. Conceito preliminar de curso. Disponível em: <http://inep.gov.br/web/guest/conceito-preliminar-de-curso-cpc-> Acesso em: 16 fev. 2019. 


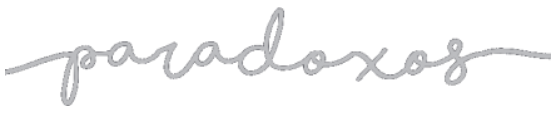

A formação audiovisual dos educadores:

A educação audiovisual nas

graduações em Pedagogia no Brasil

LIBÂNEO, José Carlos. Adeus professor, adeus professora? Novas exigências educacionais e profissão docente. 1 ed. Goiânia: Cortez Editora, 1998. 49p.

MACHADO, Eliany Salvatierra. Educomunicação e Educação Midiática: vertentes históricas de aproximação entre comunicação e educação. Revista de Estudos Universitários, Sorocaba, v. 44, n. 1, p. 53-68, jun. de 2018.

SCHEIBE, Leda. Diretrizes curriculares para o curso de Pedagogia: trajetória longa e inconclusa. Cadernos de Pesquisa, São Paulo, v. 37, n. 130, p. 43-62, jan./abr. 2007.

SOARES, Ismar De Oliveira. Educomunicação e educação midiática: vertentes históricas de aproximação entre comunicação e educação. Comunicação e Educaçãa, São Paulo, v. 19, n. 2, p. 15-26, set. de 2014.

UNIVERSIDADE DA INTEGRAÇÃO INTERNACIONAL DA LUSOFONIA AFROBRASILEIRA. Projeto Pedagógico Curricular: Licenciatura em Pedagogia. 2016.

UNIVERSIDADE DE BRASÍlIA. Projeto acadêmico do curso de Pedagogia a distância. 2011.

UNIVERSIDADE ESTADUAL DO CENTRO-OESTE DO PARANÁ. O curso de pedagogia/irati. 2019.

UNIVERSIDADE ESTADUAL PAULISTA. Criação do curso de Pedagogia no IBICLE/UNESP. 2001.

UNIVERSIDADE FEDERAL DE SÃO CARLOS. Projeto Pedagógico: Curso de Licenciatura em Pedagogia - modalidade Educação a Distância. 2010.

UNIVERSIDADE FEDERAL DE SÃO CARLOS. Projeto Pedagógico - Curso de Licenciatura em Pedagogia campus Sorocaba. 2014.

UNIVERSIDADE FEDERAL RURAL DE PERNAMBUCO. Projeto Político Pedagógico do curso de graduação em Pedagogia, licenciatura. 2006.

VELLOSO, Beatriz. Dias \& Riedweg. Rio de Janeiro: Apicuri, 2012. 152p. 